\title{
Pore Structure Characterization of Oak via X-ray Computed Tomography
}

\author{
Liangyan Guo, Hongchao Cheng, Junfeng Chen, Wentao Chen, and Jingyao Zhao * \\ The microscopic pore structure of wood is an important factor that affects its \\ macroscopic properties. In this study, an oak sample was used for pore \\ structure characterization. X-ray computed tomography imaging was carried \\ out, and the scanning results (a multi-layer two-dimensional planar image) \\ were rendered using Avizo software (a three-dimensional stereo image). A \\ digital image processing method was used to identify the characteristics of \\ the three-dimensional pore structure features and to calculate the \\ characteristic parameters, i.e., the porosity (volume/surface), pore area and \\ volume, pore size distribution, and the connectivity. The $27 \mathrm{~mm}^{3}$ oak sample \\ had the following characteristics: a pore size which ranged from $8.56 \mu \mathrm{m}$ to \\ $1262.84 \mu \mathrm{m}$; a pore volume of $1.01 \times 10^{10} \mu^{3}$; a pore area and volume \\ porosity of $1.12 \times 10^{9} \mu^{2}$ and $37.6 \%$, respectively; a surface porosity range \\ of approximately $36.1 \%$ to $39.1 \%$; a pore diameter for axial connection \\ ranging from approximately $164.57 \mu \mathrm{m}$ to $1262.84 \mu \mathrm{m}$; and had \\ corresponding proportions of the pore area and volume of approximately \\ $74.4 \%$ and $67.3 \%$, respectively. This information provided useful structural \\ data for the construction of future models.
}

Keywords: Pore structure; Porosity; Connectivity; XCT; Digital image processing

Contact information: Key Laboratory of Bio-based Material Science \& Technology (Northeast Forestry University), Ministry of Education, Harbin 150040 China;

*Corresponding author: zjy_20180328@nefu.edu.cn

\section{INTRODUCTION}

The primary reason for the large variation in the characteristics of wood can be attributed to its complex microscopic pore structure. Furthermore, the microscopic pore structure of wood is also an important factor that affects its macroscopic moisture migration, heat transfer, and mechanical properties. Therefore, from a basic research and engineering applications perspective, a systematic in-depth investigation of the structure of the wood pores was of considerable interest.

Techniques for the characterization of the pore structure of wood included mercury intrusion porosimetry (MIP), gas adsorption (GA), scanning electron microscopy (SEM), and nuclear magnetic resonance (NMR) spectroscopy (Wang and Ximing 2014).

Among these techniques, MIP and GA are the most widely used for the characterization of wood pore structures (Kojiro et al. 2009; Plötze and Niemz 2010; Chang et al. 2011; Vitas et al. 2019; Jang and Kang 2019). These methods use the nonwetting properties of either $\mathrm{Hg}$ (mercury) or gas. Information on the pore structure is obtained indirectly by detecting the total amount of $\mathrm{Hg}$ or gas that enters the porous wood. However, both techniques have some weaknesses. Due to the pressure that occurs from the mercury intrusion, a part of the pore structure is inevitably altered during the intrusion process. This results in experimental error for the tested sample. In addition, mercury is toxic, and there are safety risks associated with this method. The method destroys the 
sample, since the investigated sample cannot be reused. The gas absorption operation is based on the detection of changes in pressure and temperature. Small variations can greatly impact the measurement results, and this method can only measure the area near the entrance of the sample; this inevitably leads to experimental errors. Scanning electron microscopy has obvious advantages in terms of the qualitative characterization of the 2-D (two-dimensional) pore structure, but the possibility of quantitative analysis is limited (Fuhr et al. 2012; Blanco et al. 2017). Nuclear magnetic resonance analysis is based on the Gibbs-Thomson equation, which can measure closed cells and is easy to operate (Wang and Chang 1986; Zhou et al. 2018; Bertolini et al. 2019). However, the equation used for calculations with this method is dependent on multiple variables, and errors can easily occur. In addition, the measurement range is small, and the experimental conditions are harsh.

X-ray computed tomography (XCT) is a non-contact, non-destructive 3-D (threedimensional) microstructure characterization technique for materials. This technique can characterize samples with 3D structures at different scales. It has been successfully applied to investigate a variety of porous materials (Corigliano et al. 2017; Borges et al. 2019; Wan et al. 2019; Zhu et al. 2019). In the wood science field, researchers have used XCT to successfully observe the shrinkage behavior of wood (Taylor et al. 2013). X-ray computed tomography has also been applied to research related to wood archaeology (Stelzner and Million 2015; Maher 2020). Many sensitive wooden artifacts, e.g., wooden coffins, have been investigated via XCT in order to perform nondestructive testing. Information on the overall structure of the wood and the distribution of the medullary ray were obtained via XCT. In a study of timber rings, XCT successfully measured the internal structure of the wood and the annual ring density (Bondarenko et al. 2018). In a report on the penetration of adhesives and wood, researchers observed the penetration of the binder in the cell wall of wood via XCT. The researchers also observed the gradient of the binder penetration within the cell wall structure (Paris and Kamke 2015; Paris et al. 2015). However, there have been few reports on the usage of XCT for applications related to the investigation of the pore structure of wood, especially for pore connectivity applications.

In this work, XCT was used to determine the three-dimensional pore structure of wood. Combined with digital image processing methods, the characteristic parameters, i.e., the porosity, pore area, pore volume, pore size distribution, and connectivity were quantitatively calculated. The obtained wood pore data will be useful for pore structure reconstruction and the expansion of its applications.

\section{EXPERIMENTAL}

\section{Materials}

The oak wood samples were collected from the Greater Khingan Range forest area for investigation. The oak wood sample was cut into a cube with approximate dimensions of $3 \mathrm{~mm} \times 3 \mathrm{~mm} \times 3 \mathrm{~mm}$. The three samples used for the XCT analysis was taken from the junction between the earlywood and the latewood in the heartwood. Each surface of the sample was sanded with 1000 grit sandpaper (a particle size of approximately $25.4 \mu \mathrm{m}$ ), and then the sample was oven-dried before testing. In order to eliminate the influence of water evaporation on pore structure in the detection process, the sample was oven-dried before testing. The drying temperature was $103{ }^{\circ} \mathrm{C}$, and the time was $24 \mathrm{~h}$. 


\section{Methods}

XCT setup

The XCT setup used a nano Voxel 3000 (Sanying Precision Instruments Co., Ltd., Tianjin, China). The maximum sweep voltage and current used was $100 \mathrm{kV}$ and $200 \mu \mathrm{A}$, respectively. The flat panel detector size was $250 \mathrm{~mm} \times 200 \mathrm{~mm}$. The resolution was less than $5 \mu \mathrm{m}$, the number of physical pixel units in the detector was greater than or equal to $4800 \times 3200$, and the maximum resolution of a single two-dimensional reconstructed slice was greater than or equal to 12000 pixels $\times 12000$ pixels. The distance between the sample and the ray source, and the ray source and the detector were $7.07 \mathrm{~mm}$ and $203.20 \mathrm{~mm}$, respectively. The rotary scanning mode was used as the imaging mod, the exposure time for each image was approximately $1500 \mathrm{~ms}$, and it took approximately $90 \mathrm{~min}$ to complete a scan; a Dell workstation (Precision 7530) was used as the computer. After scanning, the data were saved in a 16-bit-raw format with an original data size of 678 voxels $\times 777$ voxels $\times 563$ voxels (the size of a single voxel was $6.9 \mu \mathrm{m}^{3}$ ).

\section{Image processing}

The image data obtained with the X-CT scan were digitized before performing image recognition and quantitative analysis. Generally, this process consisted of: (1) volume rendering; (2) image cropping; (3) noise filtering; (4) threshold segmentation; and (5) marking and quantitative analysis.

All of the above operations were performed with the 3-D visualization software Avizo (version 8.0, Thermo Fisher Scientific, Waltham, MA) and ImageJ (version 1.52a, National Institutes of Health, Bethesda, MD).

\section{RESULTS AND DISCUSSION}

\section{Characterization and Analysis Reports}

Figure 1 shows the X-CT scan of the three-dimensional pore structure and the twodimensional section (cross section, radial section, and tangential section) of an oak sample. In Fig. 1, the black represents the pores and the gray represents the matrix. This image clearly reveals the main pore structures, such as vessels, wood fibers, and wood rays.

After a preliminary quantitative analysis of the above structures, the basic parameters of the corresponding pore structures could be obtained, as shown in Table 1. It was worth noting that the X-CT measurements were able to be used to determine the basic parameters, such as the volume porosity, pore volume, and area, as well as to obtain information that could not be detected via conventional methods, e.g., the surface porosity.

Table 1. Basic Parameters of Pore Structure from Oak

\begin{tabular}{|c|c|c|c|c|c|}
\hline$r$-min $(\mu \mathrm{m})$ & $\mathrm{r}$-max $(\mu \mathrm{m})$ & $\mathrm{r}$-avg $(\mu \mathrm{m})$ & Volume $\left(\mu \mathrm{m}^{3}\right)$ & Area $\left(\mu \mathrm{m}^{2}\right)$ & Porosity $(\%)$ \\
\hline 8.56 & 1262.84 & 16.81 & $1.01 \times 10^{10}$ & $1.12 \times 10^{9}$ & 37.57 \\
\hline
\end{tabular}

Note: porosity $=V_{\text {pore }} / V_{\text {sample }}$

As shown in Table 1, the pore size range of the oak samples was approximately $8.56 \mu \mathrm{m}$ to $1260 \mu \mathrm{m}$, and the volume porosity was $37.6 \%$. The porosity obtained in this study was lower than the $44.3 \%$, which was determined via the mercury intrusion test in the study. 


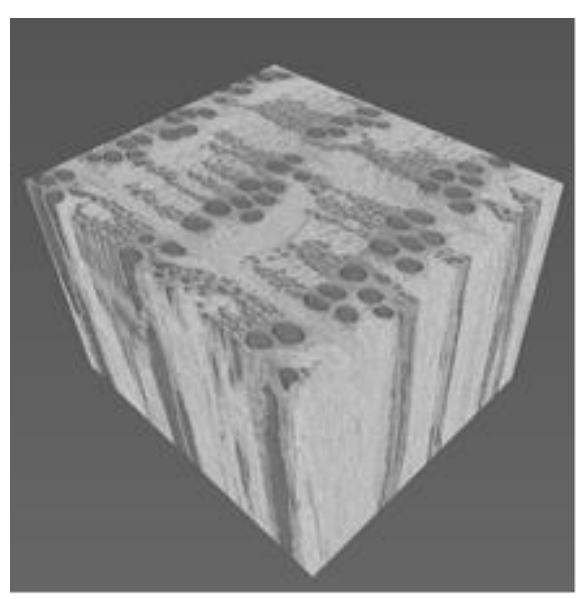

(a)

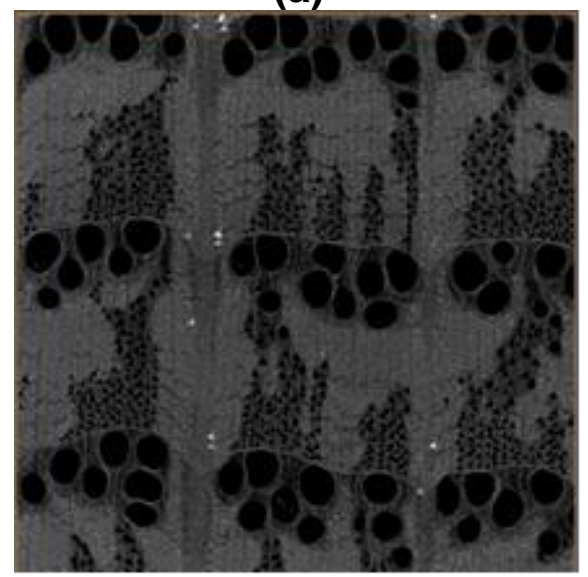

(c)

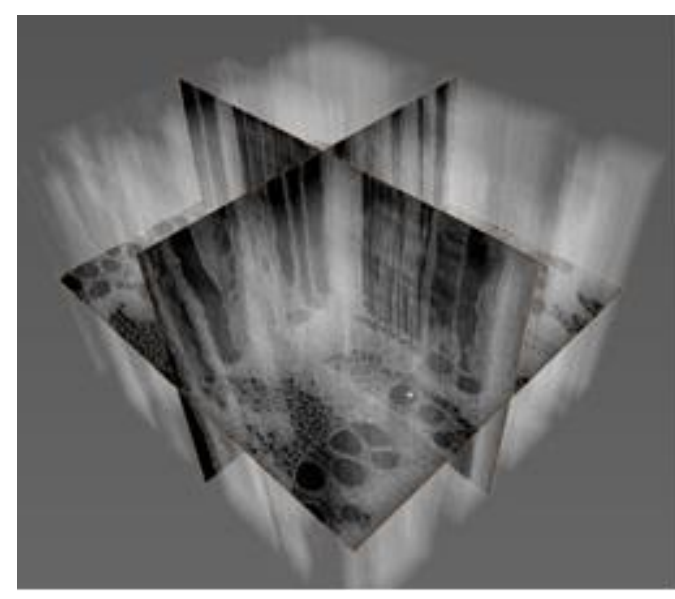

(b)

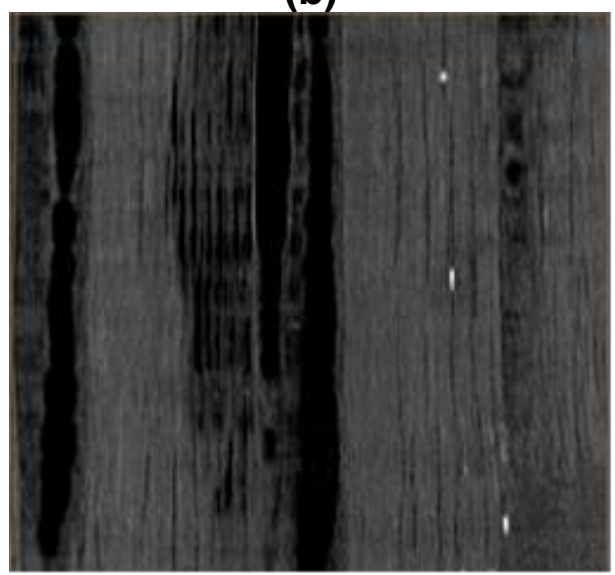

(d)

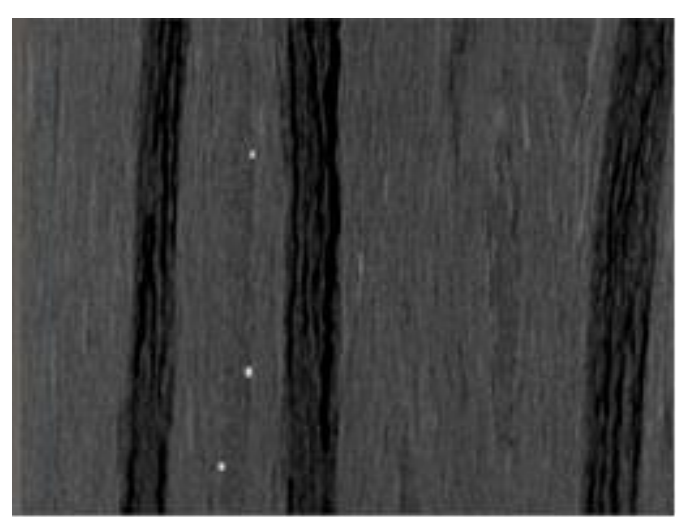

(e)

Fig. 1. The 3-D pore structure and 2-D slice image of oak wood. (a) and (b) are the 3-D pore structures, while (c), (d), and (e) are the cross, radial and tangential sections, respectively.

The sample used for this study was the same and the setup was an Auto-pore IV 9500 (Micromertitics, Atlanta, GA). This large discrepancy could have occurred due to the following three aspects. First, the resolution of XCT imaging was insufficient and only the micron scale porosity could be detected in this study; the nanometer level pores of the wood were not observable via XCT. Hence, the detected pore volume was less than the value measured via the mercury injection method. Second, the mercury intrusion method 
assumes connected cylindrical pores, so the experimentally determined porosity value is often higher than the actual value. Third, during the process of mercury intrusion, the microscopic cell wall of the wood was damaged due to the pressure of the mercury intrusion, which led to an increased amount of injected mercury. Therefore, the detection results would be artificially larger.

In addition to the volume porosity, the surface porosity of the different layers is shown in Fig. 2. Figure 2a shows the porosity distribution of each of the 513 layers, with a mean value of $37.6 \%$, a standard deviation of $0.57 \%$, and a fluctuation range of 36.0 to $39.1 \%$. As shown by Fig. 2b, there was a certain difference in the surface porosity of each layer. This could be related to the natural variability of the wood samples. The difference in the surface porosity indicated that errors could occur when using two-dimensional SEM images to measure the surface porosity, and then using these images to approximate the volume porosity.

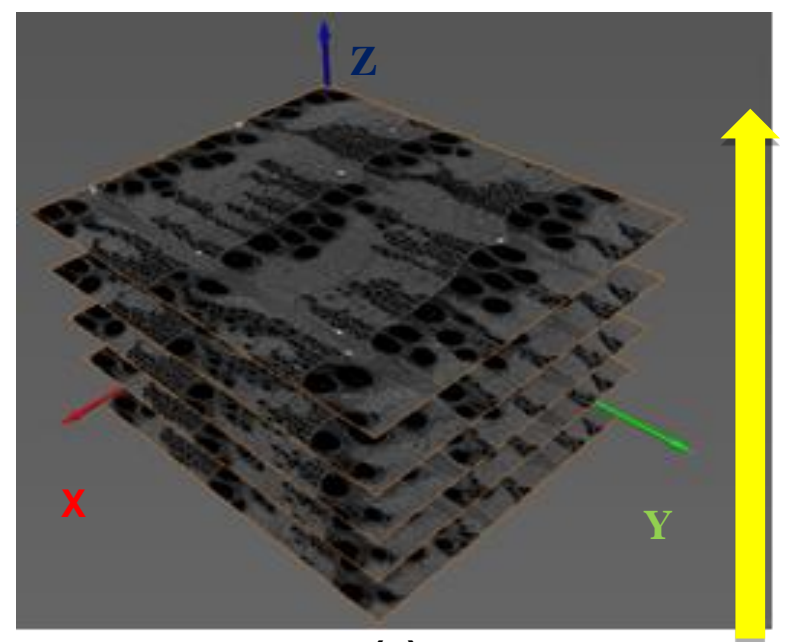

(a)

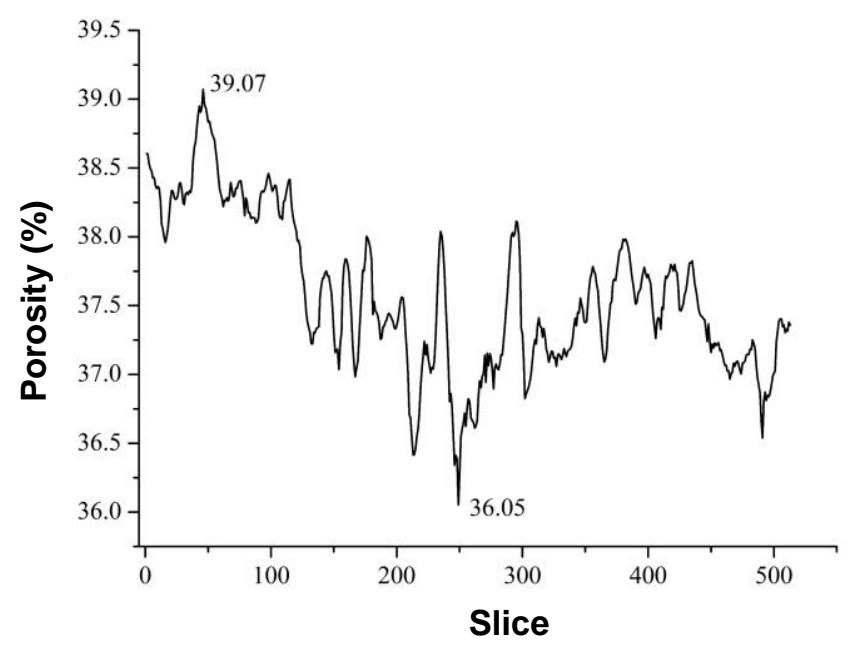

(b)

Fig. 2. Surface porosity from different slice layers. (a) slice along the $z$ axis; (b) porosity from different slice 


\section{Quantification and Analysis of the Pore Structure Parameters}

Figure 3 shows the three-dimensional pore structure that was obtained from different processing states after undergoing digital image processing, as follows: (a) was the original image; (b) was the pore and matrix image after filtering and threshold segmentation; (c) and (d) were the pore and the matrix images after separation, respectively; (e) was the pore structure of the axial connection; and (f) was the pore connectivity after screening. The pore aperture range of the axial connectivity was approximately $165 \mu \mathrm{m}$ to $1263 \mu \mathrm{m}$, and the pore area and volume ratio were approximately $74.4 \%$ and $67.3 \%$, respectively. During the detection process, only the axial pores were found to have connectivity, while the tangential and radial pores did not display connectivity. This phenomenon may be related to the definition of connectivity.

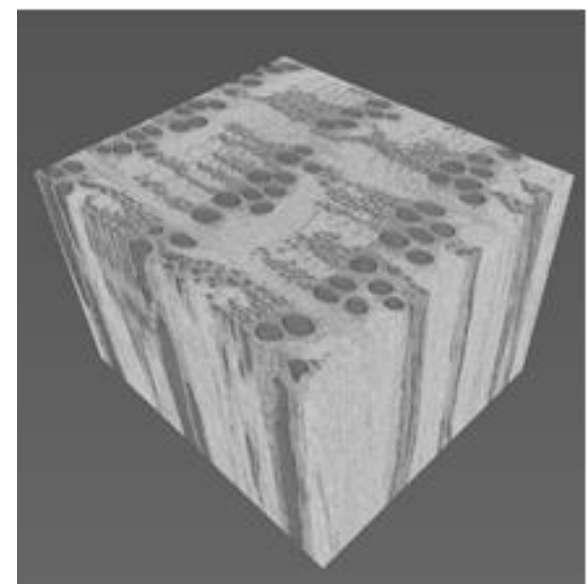

(a)

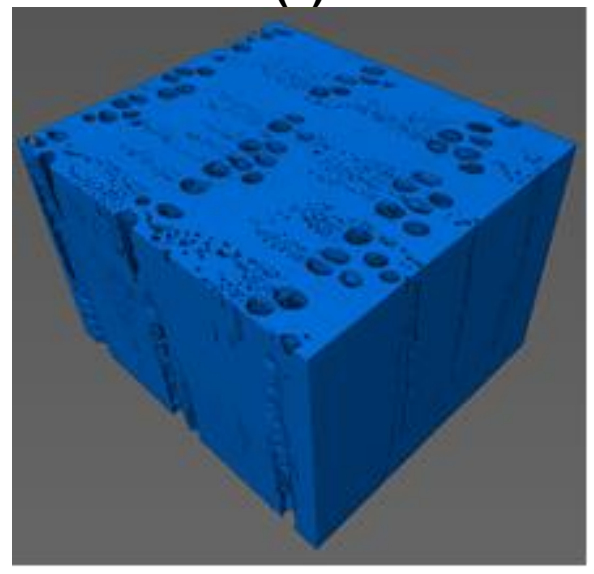

(c)

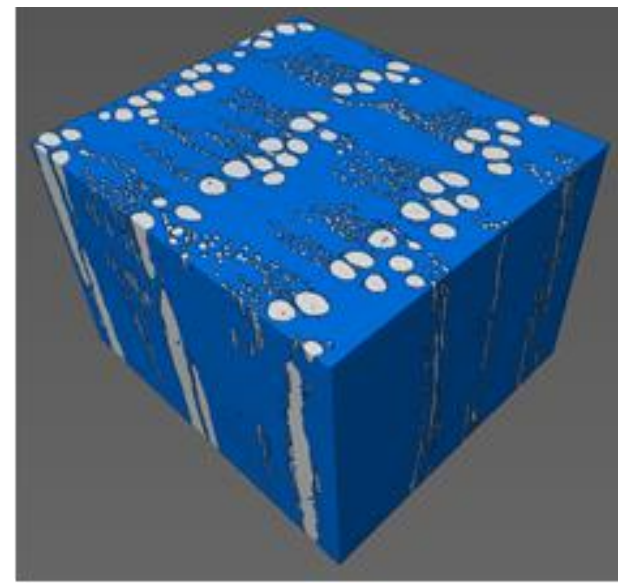

(b)

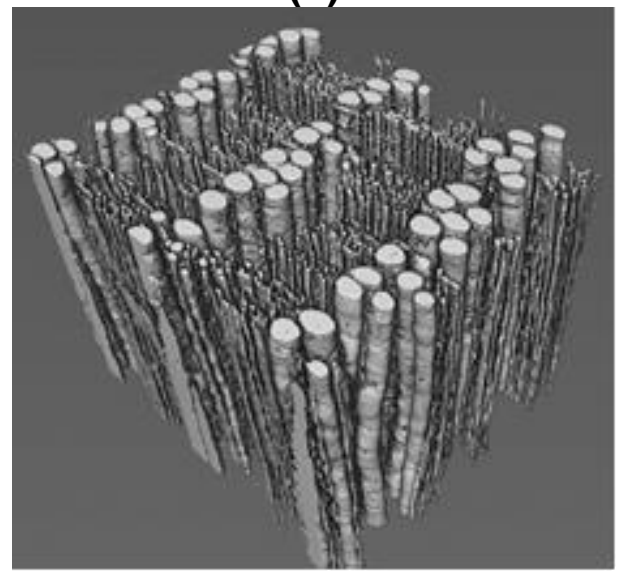

(d) 


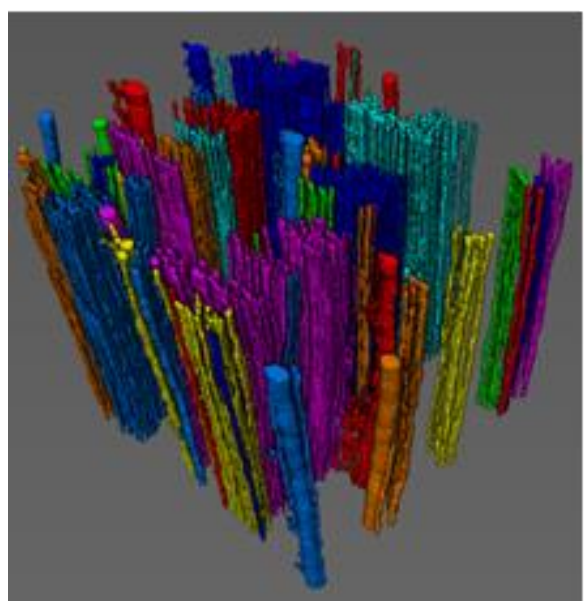

(e)

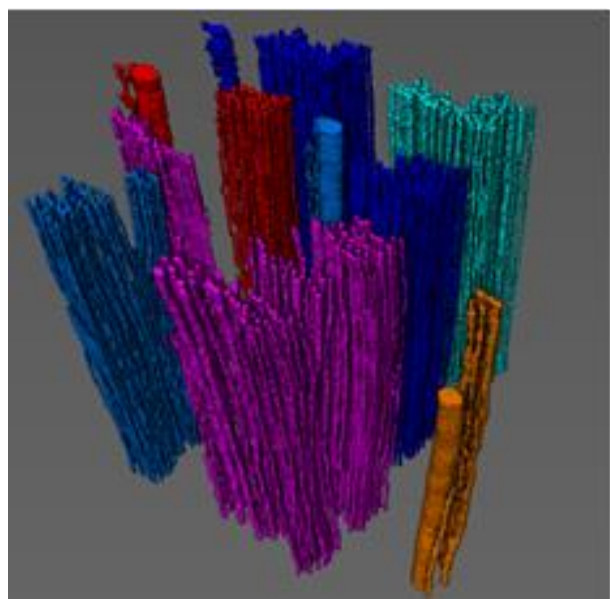

(f)

Fig. 3. The 3-D pore structure, (a) the original image, (b) the matrix and pore, (c) the matrix distribution, (d) the pore distribution, (e) the connectivity, and (f) the sieved connectivity

Broadly speaking, connectivity refers to a topological property of a space or a set. Connectivity implies a continuous process; however, in the pore structure of wood, there are very few pores that span the entire wood sample from top to bottom, and most of them are partially connected (Fig. 3b). The connectivity test in this study may have been too rigorous and formed only in axial pores, with no pores being formed in the other directions. It was shown in Fig. 3e and Fig. 3f that the transverse part showed the presence of interconnecting pores and a single color represented the same pore. Therefore, the definition of connectivity could be improved in the future, and the concept of "local connectivity" was proposed to more accurately assess and quantify pore connectivity.

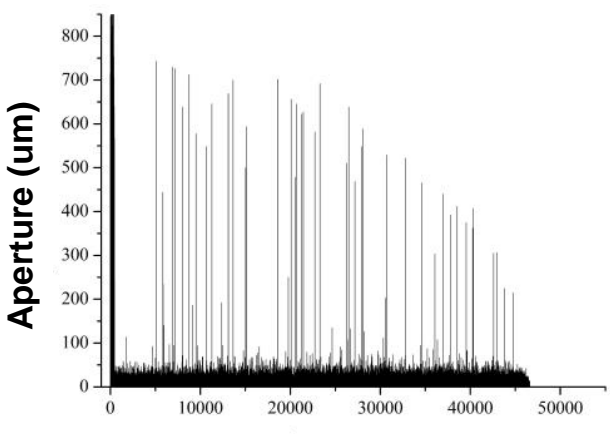

(a)

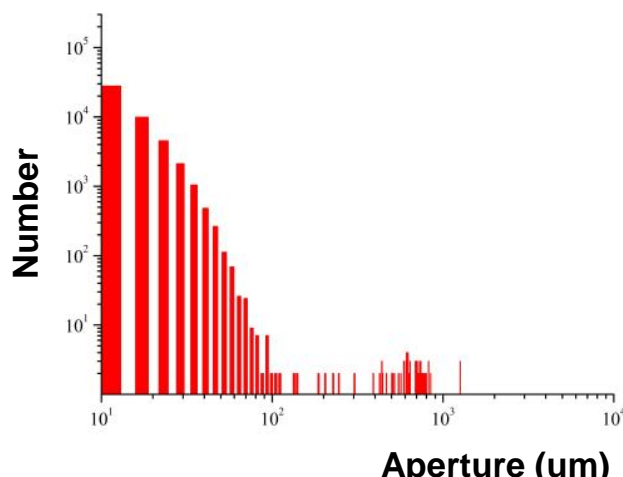

(b) 


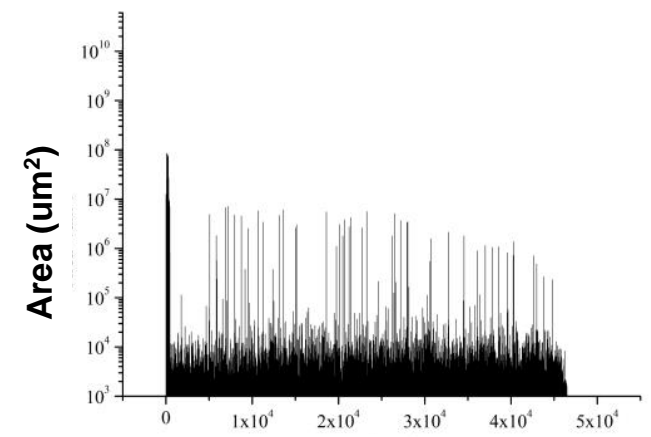

(c)

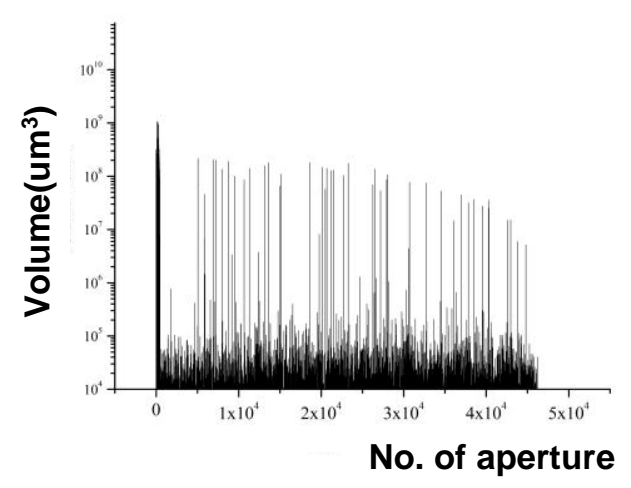

(e)

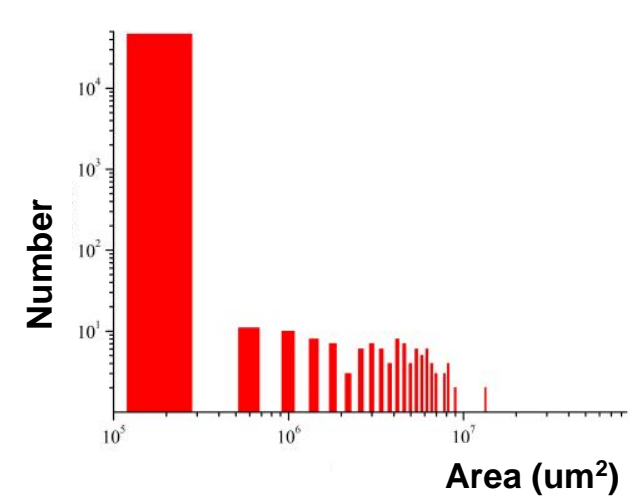

(d)

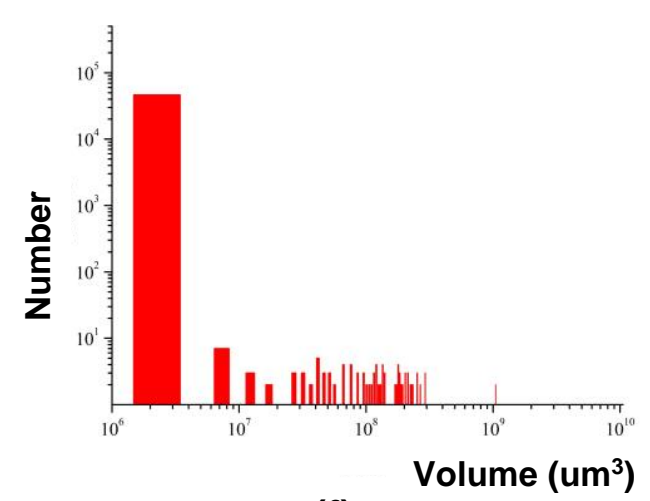

(f)

Fig. 4. Aperture, area, and volume. (a), (c), and (e) are the distributions; (b), (d), and (f) are statistics

According to the three-dimensional pore structure shown in Fig. 3, the parameters of the pore structure were quantitatively analyzed to obtain the pore diameter, pore area, and pore volume distribution information (Fig. 4).

Figures $4 \mathrm{a}, 4 \mathrm{c}$, and $4 \mathrm{e}$ showed the pore diameter, pore area, and pore volume distribution of all the pores, respectively. It was shown that the majority of the pores were small with sizes less than $100 \mu \mathrm{m}$ (primarily composed of wood fiber and wood ray tissue), and the corresponding pore area and volume were concentrated in the ranges of $2.48 \times$ $10^{4} \sim 1.99 \times 10^{5} \mu \mathrm{m}^{2}$ and $7.57 \times 10^{5} \sim 2.46 \times 10^{6} \mu \mathrm{m}^{3}$, respectively.

To more clearly see the statistical distribution of the pore diameter, pore area, and pore volume, statistical analysis was carried out on the pores. The results are shown in Fig. $4 \mathrm{~b}, 4 \mathrm{~d}$, and $4 \mathrm{f}$. As the pore size increased, the corresponding pore area and pore volume decreased. The contribution of the small pores, the pore area, and the pore volume were approximately $99.1 \%, 49.4 \%$, and $65.2 \%$, respectively. The proportion of the small pore volume was consistent with the basic characteristics of hardwood. The wood fibers, wood rays, and vessels accounted for $c a$. 50\%, 17\%, and 20\%, respectively (Liu 2012).

The above data showed that the small pores composed of wood fibers and wood rays accounted for a large proportion of the whole material and accounted for approximately $65.2 \%$ of the oak sample. However, while the vessel size was large, the number of vessels was small, accounting for $34.8 \%$ of the sample. It could be concluded that in the microscopic pore structures of wood, the pores composed of wood fibers and other structures had a large influence on the physical parameters, i.e., the macroscopic 
seepage, diffusion, and heat conduction. Therefore, for the construction of a pore equivalent model, the contribution of the macropores as well as the influence of the small pores needs to be considered. Furthermore, the quantitative results of the pore diameter distribution obtained in this study were also beneficial for simulating heat and mass transfer during the process of wood drying and heat treatment, which could greatly improve simulation accuracy and improve the experimental results.

\section{CONCLUSIONS}

1. X-ray computed tomography was demonstrated to be an effective method to detect the pore structures of wood. When combined with digital image processing, it could more quantitatively characterize the pore structures of wood.

2. X-ray computed tomography was able to obtain conventional information about the pore size distribution, porosity, pore area, and pore volume; as well as obtain information that was not accessible via other detection methods, e.g., the threedimensional structure and the pore connectivity. This information provided useful structural data for the construction of future models. However, due to resolution limitations, this study only investigated the wood structure on a micrometer scale.

\section{ACKNOWLEDGMENTS}

This work was financially supported by the National Innovation Training Program for College Students of Northeast Forestry University (Grant No. 201910225018) and the National Natural Science Foundation of China (Grant No. 31901242).

\section{REFERENCES CITED}

Bertolini, M. D. S., Morais, C. A. G. D., Christoforo, A. L., Bertoli, S. R., Santos, W. N. D., and Lahr, F. A. R. (2019). "Acoustic absorption and thermal insulation of wood panels: Influence of porosity," BioResources 14(2), 3746-3757. DOI: 10.15376/biores.14.2.3746-3757

Blanco, M. V. D., Fischer, E. J., and Cabane, E. (2017). "Underwater superoleophobic wood cross sections for efficient oil/water separation," Advanced Materials Interfaces 4(21), 103-110. DOI: 10.1002/admi.201700584

Bondarenko, S. L., Batranin, A. V., Smirnov, S. V., and Stuchebrov, S. G. (2018). "X-ray imaging and computed tomography of conifer tree rings for climatological purposes," in: International Conference and Early Career Scientists School on Environmental Observations, Modeling and Information Systems, 5-11 July, Tomsk, Russia. DOI:10.1088/1755-1315/211/1/012044

Borges, J. A. R., Pires, L. F., Cássaro, F. A. M., Auler, A. C., Rosa, J. A., Heck, R. J., and Roque, W. L. (2019). "X-ray computed tomography for assessing the effect of tillage systems on topsoil morphological attributes," Soil and Tillage Research 189, 25-35.

DOI:10.1016/j.still.2018.12.019 
Chang, S., Jinbo, H., Bruno, C., and Françoise, Q. (2011). "Pore structure characterization of poplar tension wood by nitrogen adsorption-desorption method," Scientia Silvae Sinicae 47(10), 137-143. DOI: 10.11707/j.1001-7488.20111021

Corigliano, P., Crupi, V., Epasto, G., Guglielmino, E., Maugeri, N., and Marinò, A. (2017). "Experimental and theoretical analyses of Iroko wood laminates," Composites Part B: Engineering 112, 251-264. DOI: 10.1016/j.compositesb.2016.12.049

Fuhr, M. J., Stührk, C., Münch, B., Schwarze, F. W. M. R., and Schubert, M. (2012). "Automated quantification of the impact of the wood-decay fungus Physisporinus vitreus on the cell wall structure of Norway spruce by tomographic microscopy," Wood Science and Technology 46(4), 769-779. DOI: 10.1007/s00226-011-0442-y

Jang, E. S., and Kang, C. W. (2019). "Changes in gas permeability and pore structure of wood under heat treating temperature conditions," Journal of Wood Science 65(1), 19. DOI: $10.1186 / \mathrm{s} 10086-019-1815-3$

Kojiro, K., Miki, T., Sugimoto, H., Nakajima, M., and Kanayama, K. (2009). "Micropores and mesopores in the cell wall of dry wood," Journal of Wood Science 56(2), 107-111. DOI: 10.1007/s 10086-009-1063-z

Liu, Y. X. (2012). Wood Science, China Forestry Publishing House, Beijing, China.

Maher, M. A. (2020). "X-ray computed tomography of a late period falcon bronze coffin," Radiation Physics and Chemistry 166, 108475. DOI: 10.1016/j.radphyschem.2019.108475

Paris, J. L., and Kamke, F. A. (2015). "Quantitative wood-adhesive penetration with Xray computed tomography," International Journal of Adhesion and Adhesives 61, 7180. DOI: $10.1016 /$ j.ijadhadh.2015.05.006

Paris, J. L., Kamke, F. A., and Xiao, X. (2015). "X-ray computed tomography of woodadhesive bondlines: Attenuation and phase-contrast effects," Wood Science and Technology 49(6), 1185-1208. DOI: 10.1007/s00226-015-0750-8

Plötze, M., and Niemz, P. (2011). "Porosity and pore size distribution of different wood types as determined by mercury intrusion porosimetry," European Journal of Wood and Wood Products 69(4), 649-657. DOI: 10.1007/s00107-010-0504-0

Stelzner, J., and Million, S. (2015). "X-ray computed tomography for the anatomical and dendrochronological analysis of archaeological wood," Journal of Archaeological Science 55, 188-196. DOI: 10.1016/j.jas.2014.12.015

Taylor, A., Plank, B., Standfest, G., and Petutschnigg, A. (2012). "Beech wood shrinkage observed at the micro-scale by a time series of X-ray computed tomographs ( $\mu$ XCT)," Holzforschung 67(2), 201-205. DOI: 10.1515/hf-2012-0100

Vitas, S., Segmehl, J. S., Burgert, I., and Cabane, E. (2019). "Porosity and pore size distribution of native and delignified beech wood determined by mercury intrusion porosimetry," Materials 12(3), 416-429. DOI:10.3390/ma12030416

Wan, F., Pirzada, T. J., Liu, R., Wang, Y., Zhang, C., and Marrow, T. J. (2019). "Microstructure characterization by X-ray computed tomography of C/C-Sic ceramic composites fabricated with different carbon fiber architectures," Applied Composite Materials 26(4), 1247-1260. DOI: 10.1007/s10443-019-09778-2

Wang, P. C., and Chang, S. J. (1986). "Nuclear magnetic resonance imaging of wood," Wood and Fiber Science 18(2), 308-314.

Wang, Z., and Ximing, W. (2014). "Research progress of multi-scale pore structure and characterization methods of wood," Scientia Silvae Sinicae 50(10), 123-133.

Zhou, M., Caré, S., Courtier-Murias, D., Faure, P., Rodts, S., and Coussot, P. (2018). "Magnetic resonance imaging evidences of the impact of water sorption on hardwood 
capillary imbibition dynamics," Wood Science and Technology 52(4), 929-955. DOI: 10.1007/s00226-018-1017-y

Zhu, L., Dang, F., Xue, Y., Ding, W., and Zhang, L. (2019). “Analysis of microstructural damage evolution of concrete through coupled X-ray computed tomography and gray-level co-occurrence matrices method," Construction and Building Materials 224, 534-550. DOI: 10.1016/j.conbuildmat.2019.07.007

Article submitted: December 16, 2019; Peer review completed: February 22, 2020;

Revised version received: March 8, 2020; Accepted: March 11, 2020; Published: March 16, 2020.

DOI: 10.15376/biores.15.2.3053-3063 